\title{
Contrarrestar el estigma hacia las personas con esquizofrenia en el ámbito sanitario: una experiencia piloto en una muestra de estudiantes italianos de medicina
}

\author{
Lorenza Magliano ${ }^{1 *}$, John Read ${ }^{2}$, Melania Patalano ${ }^{3}$, Alessandra Sagliocchi ${ }^{3}$, Nicoletta Oli- \\ viero $^{3}$, Antonio D'Ambrosio ${ }^{3}$, Federica Campitiello ${ }^{3}$, Antonella Zaccaro ${ }^{3}$, Lorenzo Guizzaro ${ }^{3}$ y $^{2}$ \\ Ferdinando Cerrato ${ }^{3}$
}

${ }^{1}$ Dipartimento di Psicologia, Seconda Università di Napoli ${ }^{2}$ School of Psychology, University of Auckland, Nueva Zelanda ${ }^{3}$ Facoltà di Medicina e Facoltà di Psicologia, Seconda Università di Napoli.

(Recibido 14 Septiembre, 2012; Aceptado 23 Octubre, 2012)

RESUMEN: El artículo parte de la preocupación por los efectos negativos, constatados en múltiples publicaciones internacionales, que el estigma tiene sobre la calidad de la atención que reciben las personas con esquizofrenia en el ámbito de los servicios sanitarios y en relación con su salud física, frecuentemente deteriorada. Como consecuencia del desconocimiento sobre las causas y características de esta enfermedad y las ideas erróneas que muchos profesionales comparten con la población general sobre la peligrosidad e incurabilidad de las personas afectadas por este trastorno, son habitualmente objeto de falta de respeto y mala atención en los servicios sanitarios generales.

Un momento importante para mejorar la información y corregir las ideas erróneas es el periodo de formación de los profesionales y a ese respecto se presentan los primeros resultados de una experiencia piloto, desarrollada entre estudiantes de Medicina de una Universidad italiana, consistente en la inclusión de un curso breve sobre el estigma y la esquizofrenia.

Aunque los resultados deben ser confirmados por otros estudios, permiten constatar los evidentes cambios positivos que se producen en el conocimiento y las actitudes de los estudiantes con una breve intervención educativa, asociada a la participación de usuarios, confirmando también otros estudios internacionales. Palabras clave: estigma, esquizofrenia, profesionales sanitarios

To counteract stigma towards people with schizophrenia in the health sector: a pilot experience in a sample of Italian students of medicine

\begin{abstract}
The article is based of the concern about the negative effects, found in many international publications, that stigma has on the quality of care that people with schizophrenia receive in the field of health services, in connection with their physical health, often deteriorated. As a result of the lack of knowledge about the causes and characteristics of this disease and the misconceptions that many professionals share with the general population about the dangerousness and lack of recovery of the people affected by this disorder, they are usually subject of lack of respect and poor care into general health services.
\end{abstract}


Very important to improve the information and correct erroneous attitudes is the period of training of professionals and in this regard are presented the first results of a pilot project, developed between students of Medicine of an Italian University, consisting in a brief course on stigma and schizophrenia.

Although the results must be confirmed by other studies, they noted the evident positive changes occurring in the knowledge and attitudes of students with a brief educational intervention, associated with the participation of users, also confirming other international studies.

Keywords: stigma, schizophrenia, health professionals

\section{CONSIDERACIONES SOBRE EL ESTIGMA Y LA ESQUIZOFRENIA EN EL ÁMBITO SANITARIO}

"No hay salud sin salud mental". Este es el título de un editorial de la revista The Lancet (Lancet, 2011) que subrayaba la deficiente salud física de las personas con trastornos mentales y la necesidad de mejorar la calidad de la atención médica que reciben. De hecho, cuando una persona con un diagnóstico psiquiátrico sufre una patología física, su capacidad de acceder a los servicios sanitarios especializados y de recibir atención eficaz y oportuna, disminuye como resultado del "status" de "enfermo mental" (Sartorius, 2002; Aydin et al., 2003; Schulze y Angermeyer, 2003; Patel, 2004; Nordt et al., 2006; Grausgruber et al., 2007; Jones et al. 2008; Mengod, 2007).

Es también por ese motivo que, en las personas con problemas psíquicos, las enfermedades físicas se cronifican con más frecuencia y el sufrimiento que comportan se añade al de las enfermedades mentales contribuyendo a agravarlas y a prolongar su duración.

Este es otro efecto del estigma (Magliano et al., 2004a; Angermeyer y Dietrich, 2006; Read et al., 2006; Jorm y Oh, 2009; Angermeyer et al., 2010; Angermeyer et al., 2011), una "incurable" segunda enfermedad que, a pesar de los esfuerzos y las campañas de sensibilización, acompaña a los trastornos mentales y a menudo persiste incluso cuando estos se han curado (Wahl, 1999; Angermeyer y Matschinger, 2004). Esta situación es aún más difícil en el caso de la esquizofrenia, una de las patologías psiquiátricas con mayor estigma personal y social (Cormack y Furnham 1998; Readet al., 2006; Read et al., en prensa).

Las personas con esquizofrenia tienen, sin embargo, un alto riesgo de mortalidad prematura por enfermedades cardiovasculares, metabólicas y tumorales (Casey, 2005; Newcomer, 2007; Howard et al., 2010). Una situación ligada verosímilmente al tratamiento prolongado con antipsicóticos, a los estilos de vida de riesgo (tabaquismo, abuso de substancias, deficiente alimentación) y a la menor atención reservada en el ámbito sanitario a las personas afectadas por este trastorno mental (Thornicroft et al., 2007).

En un intento de aumentar la aceptación social de las personas con este diagnóstico, paralelamente al cambio hacia un modelo más estrechamente médicobiológico del sufrimiento psíquico, se ha presentado la esquizofrenia a la gente 
común como "una enfermedad como las otras" (Read et al., 2006; Pescosolido et al., 2010). En particular, se ha dado gran importancia a los aspectos genéticos y se ha prestado menos atención a los psicosociales implicados en la génesis de esta patología (Luchins, 2004; Lee et al., 2006).

Sin embargo, la adopción de un modelo de biogenético no ha aumentado de hecho la comprensión de los "otros" en relación con las personas con este diagnóstico. Por el contrario, en los últimos años, los estudios poblacionales han puesto de manifiesto que han aumentado los prejuicios relativos a los "esquizofrénicos", así como la distancia social hacia ellos (Read et al., 2006). Si de hecho, por una parte, estas personas son menos culpabilizadas por la opinión pública y por sus familiares por las manifestaciones clínicas de su patología, por otra, son vistas todavía más como impredecibles y peligrosas, en tanto que incapaces de controlar comportamientos asociados a causas genéticas "inmutables". El modelo biogenético ha reforzado también la convicción, tanto entre la gente común como entre los profesionales sanitarios, de que la esquizofrenia es una patología ineluctablemente crónica, cuyos síntomas requieren un control farmacológico durante toda su vida (Readet al., en prensa).

En el ámbito psiquiátrico, también como consecuencia del efecto estigmatizante de esta etiqueta diagnóstica, rara vez las personas con esquizofrenia y sus familias reciben la información médica que querrían tener sobre la naturaleza y las manifestaciones de esta enfermedad y sobre la posibilidad de una recuperación (Magliano et al., 2004a; Magliano et al., 2008). De hecho, los Psiquiatras a menudo creen que hablar de este trastorno es desmoralizador para la persona a la que se le aplica, que hablar de recuperación alimenta falsas esperanzas y que informar a los usuarios sobre los efectos secundarios de los psicofármacos aumenta su desconfianza hacia estas sustancias (Magliano et al., 2004a; 2004b).

Aunque el estigma hacia las personas con esquizofrenia en la población general está ampliamente documentado, son escasas las informaciones sobre este tema en el campo médico. Los datos disponibles sugieren que los profesionales sanitarios no son inmunes a compartir con la gente común estereotipos y prejuicios referidos a las personas con esquizofrenia (Sartorius, 2002; Aydin et al., 2003; Magliano et al., 2004a; Patel, 2004; Nordt et al., 2006; Grausgruber et al., 2007) y que sus actitudes hacia quienes reciben este diagnóstico contribuyen a la deficiente salud física de estos pacientes (Jones et al., 2008).

Imprevisibles, peligrosos, afectados por una enfermedad incurable, incapaces de participar activamente en las decisiones sobre su propia vida y su propia salud. Es así como los trabajadores sanitarios consideran frecuentemente a las personas diagnosticadas de esquizofrenia. Estas creencias se traducen en una escasa atención dedicada por los médicos a los trastornos físicos de que se quejan estos pacientes, con tendencia a interpretar los síntomas de sufrimiento físico como signos de patología mental (Schulze y Angermeyer, 2003), y en una menor precisión diagnóstica y terapéutica. Es además común que estos pacientes sean tratados con menos respeto que los otros en los servicios médicos y quirúrgicos y transferidos a departamentos psiquiátricos tan pronto como sea 
posible, en razón del diagnóstico de esquizofrenia (Schulze y Angermeyer, 2003; Jones et al., 2008). Los trabajadores sanitarios usan también a menudo palabras estigmatizantes y términos ofensivos en relación con estas personas, tendiendo a identificarlos con su enfermedad ("los esquizofrénicos") o a considerarles genéricamente «locos». El personal sanitario es también muchas veces reacio a hacerse cargo de las emergencias médicas y quirúrgicas cuando es llamado a intervenir como consecuencia de gestos autolesivos relacionados con la presencia de una enfermedad mental. En los pacientes con esquizofrenia, la percepción de la desconfianza hacia ellos por parte de los médicos y trabajadores sanitarios en general, aumenta la dificultad para expresar sus propios problemas de salud por el temor, fundado, a no ser tomado en serio (Schulze y Angermeyer, 2003; Thornicroft, 2007). No sorprende, por tanto, que dada la actitud negativa hacia ellos de muchos trabajadores sanitarios, los "esquizofrénicos" tengan dificultades para acceder a una atención eficaz y a recibirla sin demora.

Una clase particular de profesionales de la salud está representada por los alumnos de la Facultad de Medicina (Llerena et al., 2002; Naeem et al., 2006; $\mathrm{Ng}$ et al., 2011). Estos estudiantes, por el contexto y por su papel de futuros médicos, representan una categoría de elección para las intervenciones que buscan la reducción del estigma en el ámbito sanitario. Los datos disponibles sobre las opiniones de los estudiantes de medicina con respecto a las enfermedades mentales ponen de manifiesto que comparten con el personal sanitario diversos prejuicios acerca de los pacientes con esquizofrenia y cómo la adhesión a un modelo biogenético y el uso de una etiqueta diagnóstica se asocian también a un aumento de la distancia social hacia estos usuarios (Magliano et al., en prensa; 2011). Los estudios muestran que los estudiantes de medicina consideran peligrosas a las personas con esquizofrenia en un porcentaje que va desde el $26 \%$ al $78 \%$, impredecibles en un porcentaje entre el $71 \%$ y el $85 \%$ y afectados por una enfermedad incurable entre un $4 \%$ y un 21\% (Ogunsemiet al., 2008; Arvaniti et al., 2009; Fernando et al., 2010; Magliano et al., 2011; Economou et al., 2012).

Otros estudios, que han examinado las actitudes de los estudiantes de medicina en diferentes etapas de su proceso de formación, han puesto también de manifiesto cómo en el trienio preclínico los estudiantes son más similares a la gente común en la interpretación de la esquizofrenia según un modelo causal psicosocial, mientras que en el trienio clínico se aproximan mayoritariamente al modelo biomédico, más común entre los trabajadores sanitarios. La adquisición de habilidades diagnósticas y terapéuticas se asocia con frecuencia a una mayor confianza en las terapias disponibles para el tratamiento de esta patología, pero no siempre a una reducción de los prejuicios de incurabilidad e imprevisibilidad de los «esquizofrénicos», a una mayor atención al contexto psicosocial de la vida de estas personas (Ay et al., 2006; Magliano et al., in press).

Desde el convencimiento de que mejorar la salud de las personas con esquizofrenia no puede prescindir de un trabajo de educación y sensibilización sobre el estigma durante la formación de los futuros profesionales sanitarios, en 2010, se estableció en la Facultad de Medicina de la Segunda Universidad de Nápoles 
un grupo de trabajo sobre el estigma y la esquizofrenia en el que participaron estudiantes bajo la coordinación de una profesora. El laboratorio, que luego se extendió a los estudiantes de la Facultad de Psicología de la misma Universidad, tenía el objetivo de estudiar las opiniones y actitudes de los futuros profesionales sanitarios hacia las personas con trastornos mentales, de estimular el debate entre estudiantes de diferentes disciplinas de las áreas sociosanitarias sobre los derechos de los pacientes y las intervenciones basadas en la evidencia y de promover iniciativas para contrarrestar el estigma en el ámbito sanitario.

Una primera encuesta realizada por el grupo de trabajo sobre 194 estudiantes de los dos últimos años de los estudios de medicina, puso de manifiesto que la herencia es el factor causal más frecuentemente referido por los estudiantes en el origen de la esquizofrenia (81\%), seguido por el estrés $(69 \%)$, el consumo de drogas $(45 \%)$ y el trauma psicológico (45\%). Además, el $76 \%$ de los estudiantes se manifestó escéptico sobre la posibilidad de recuperación de esta enfermedad, mientras que el $86 \%$ estaba convencido, totalmente o en parte, de que las personas con esquizofrenia son impredecibles, y el $73 \%$ de que pueden ser peligrosas. Este primer estudio puso tambiénde relieve que la atribución de la etiqueta diagnóstica de esquizofrenia a un caso clínico y la identificación de la herencia entre sus causas, se asociaban con un mayor pesimismo de los estudiantes sobre la posibilidad de recuperacióny a una mayor distancia social hacia las personas con este diagnóstico. Además, la atribución de la esquizofrenia a causas hereditarias se asociaba a una percepción más arraigada de la imprevisibilidad atribuida a los “esquizofrénicos” (Magliano et al., 2011).

Basandose en los resultados de este estudio, el grupo de trabajo se preguntó si las opiniones de los estudiantes se modificarían en el curso de los estudios de medicina y si la adquisición de competencias clínicas mejoraría sus actitudes hacia estas personas. La encuesta se repitió en una muestra de 187 estudiantes del primer año de medicina y las dos muestras fueron comparadas con respecto a las actitudes y a las explicaciones causales (Magliano et al., in press).

Los resultados de este segundo estudio mostraron una diferencia significativa en las opiniones entre los alumnos del primero y el último año de estudio, con un cambio desde un modelo interpretativo más psicosocial a uno más estrechamente biológico. En particular, los estudiantes cercanos a la graduación resultaron atribuir más relevancia a los factores genéticos (del 16\% en los estudiantes del primer año al $45 \%$ de los estudiantes del $5^{\circ}$ o $6^{\circ}$ año) y menor importancia a los traumas psicológicos en la génesis de este trastorno (del 76\% al 45\%). Además, en comparación con los estudiantes de primer año de medicina, los del $5^{\circ}$ y $6^{\circ}$ año se caracterizaron por una mayor distancia social percibida hacia las personas con esquizofrenia.

Sobre la base de estos hallazgos, nuestro grupo de trabajo decidió desarrollar un módulo de formación, dirigido a los estudiantes del trienio clínico, sobre «Prejuicios y evidencias científicas de la peligrosidad social y la incurabilidad de la esquizofrenia» y comprobar su efecto a corto plazo sobre los participantes.

En la siguiente sección de este artículo se describen el método y el contenido 
de esta iniciativa piloto y se presentan algunos resultados preliminares

\section{EL MÓDULO FORMATIVO SOBRE "PREJUICIOS Y EVIDENCIAS CIEN- TÍFICAS SOBRE LA PELIGROSIDAD SOCIAL Y LA INCURABILIDAD DE LA ESQUIZOFRENIA"}

\section{Método}

La iniciativa se dirigió a estudiantes de $4^{\circ}, 5^{\circ}$ y $6^{\circ}$ año de Licenciatura en Medicina y Cirugía de la Segunda Universidad de Nápoles. En Italia, los estudios de medicina tienen una duración de 6 años e incluyen materias obligatorias y materias optativas que proporcionan los créditos formativos necesarios para conseguir la Licenciatura. El modulo sobre "Prejuicios y evidencias científicas de la peligrosidad social y la incurabilidad de la esquizofrenia"estaba incluido entre las materias optativas y se desarrolló en noviembre de 2011.

El módulo preveía dos sesiones con una duración de 3 horas cada una y con un intervalo de una semana. Todos los estudiantes que hicieron la solicitud, a través del correo electrónico, fueron admitidos al mismo. Al comienzo de la primera sesión y al final de la segunda, los participantes debían responder al Cuestionario sobre las Opiniones con respecto a los trastornos mentales (CO), cumplimentado de forma anónima. El CO es un instrumento autoaplicable que explora las opiniones sobre la enfermedad mental en 4 áreas principales: 1) la utilidad de los tratamientos farmacológicos y psicosociales y el derecho a la información sobre los trastornos mentales y los tratamientos de los pacientes y sus familiares; 2) las causas de los trastornos mentales; 3) los derechos civiles y afectivos de las personas con trastornos mentales; y 4) la distancia social hacia estas personas y su supuesta imprevisibilidad y peligrosidad. Para responder a las preguntas, se pidió a los estudiantes que tomasen como referencia un caso tipo ("viñeta"), unido al $\mathrm{CO}$, que remitía a un diagnóstico de esquizofrenia según la ICD 10.

\section{Contenidos y modalidades de aplicación de las sesiones}

Con respecto al contenido del modulo, el grupo de trabajo llevó a cabo un análisis preliminar de la literatura sobre el estigma y definió a partir de ella un conjunto de temas a tratar en las dos sesiones. El siguiente cuadro recoge dichos temas. 


\begin{tabular}{|l|l|}
\hline \multicolumn{1}{|c|}{ Primera Sesión } & \multicolumn{1}{c|}{ Segunda Sesión } \\
\hline $\begin{array}{l}\text { Definiciones de los estereotipos, } \\
\text { prejuicios y discriminación. }\end{array}$ & $\begin{array}{l}\text { Evidencia científica sobre la } \\
\text { peligrosidad social en las minorías } \\
\text { "en riesgo" }\end{array}$ \\
\hline $\begin{array}{l}\text { Supuestos teóricos, estudios y } \\
\text { testimonios sobre el estigma y sus } \\
\text { efectos }\end{array}$ & $\begin{array}{l}\text { Evidencia científica sobre la } \\
\text { peligrosidad social de las personas } \\
\text { con trastornos mentales }\end{array}$ \\
\hline $\begin{array}{l}\text { Estigma y los medios de } \\
\text { comunicación }\end{array}$ & $\begin{array}{l}\text { Evidencia científica sobre la } \\
\text { peligrosidad social de las personas } \\
\text { con esquizofrenia }\end{array}$ \\
\hline Estigma y enfermedad mental & $\begin{array}{l}\text { El significado subjetivo y objetivo } \\
\text { de la recuperación de } \\
\text { enfermedades mentales }\end{array}$ \\
\hline Estigma y esquizofrenia & $\begin{array}{l}\text { Evidencia científica sobre la } \\
\text { recuperación de la esquizofrenia }\end{array}$ \\
\hline El estigma en el ámbito sanitario & $\begin{array}{l}\text { Servicios orientados a la } \\
\text { recuperación y el empoderamiento }\end{array}$ \\
\hline
\end{tabular}

Figura 1. Contenido de las dos sesiones del módulo sobre "Prejuicios y evidencias científicas de la peligrosidad social y la incurabilidad de la esquizofrenia".

Cada miembro del grupo de trabajo profundizó sucesivamente en un aspecto especifico de los que se iban a abordar durante el modulo y obtuvo material científico y de sensibilización, utilizando Publimed y los principales buscadores genéricos de bibliografía. El material utilizado (que se refiere al final de la sección de bibliografía) fue analizado por todo el grupo de trabajo y de manera conjunta se decidió como presentarlo durante la iniciativa educativa.

Desde el principio surgió la necesidad de organizar las dos sesiones implicando en ellas a usuarios con trastornos mentales y utilizando, junto con el material científico, sus testimonios.

Se invitó por tanto a participar en la actividad, en calidad de "expertos", a 4 personas con trastornos mentales. Los 4 “ expertos" enviaron una grabación de audio de su experiencia, o un escrito. En este último caso, el texto fue grabado por un estudiante antes de la clase para poder escucharlo en el aula. Las experiencias personales se referían al desarrollo de la esquizofrenia y la recuperación, a la discriminación experimentada en el ámbito sanitario, al empoderamiento y las estrategias con que afrontar la enfermedad mental y al impacto de los medios de comunicación en su tratamiento de las noticias sobre la vida de los usuarios con trastornos mentales.

Cada tema fue tratado haciendo referencia a la literatura internacional y haciendo hincapié en los puntos clave con una viñeta, un "spot" o un video de una 
campaña de sensibilización. En particular se utilizaron: un video producido por la BBC sobre el estigma y la esquizofrenia, un video de sensibilización sobre los prejuicios relativos a los homosexuales y un video de la campaña "Change your mind" ("cambia tu mente") realizada en el Reino Unido (Ver la sección "Material para el curso"). Los estudiantes del grupo de trabajo ayudaron a la profesora en la realización de las sesiones, presentando a sus compañeros participantes algunos temas en los que habían profundizado.

\section{RESULTADOS PRELIMINARES}

A las dos reuniones asistieron 112 estudiantes. Los participantes eran en un $50 \%$ de sexo femenino, con una edad media de 25 años (desviación típica de 4.3 ) y un $98 \%$ solteros.

En la evaluación inicial con el $\mathrm{CO}$, los factores más frecuentemente señalados por los estudiantes entre las causas de la esquizofrenia fueron la herencia (71\%), un desequilibrio químico (61\%), los traumas psicológicos (55\%), el consumo de drogas (52\%) y el estrés (37\%). El 68\% de los estudiantes era pesimista, en todo o en parte, sobre la posibilidad de que las personas con esquizofrenia pudieran recuperarse, mientras que el $68 \%$ eran escéptico sobre la utilidad de los psicofármacos y el $58 \%$ sobre la de las intervenciones psicosociales. Además, el 30\% estaba plenamente convencido de que las personas con diagnóstico de esquizofrenia eran impredecibles, el $51 \%$ que eran peligrosas para los otros y el $63 \%$ que lo eran para sí mismos.

Las respuestas al cuestionario, administrado al final de la segunda sesión, mostraron diferencias estadísticamente significativas en la opinión de los estudiantes sobre la esquizofrenia en comparación con la evaluación inicial. En particular, el estrés fue señalado entre las causas por el $83 \%$ de los estudiantes $(\mathrm{p}<$. $0001)$ y el trauma por el 78 por ciento $(p<.0001)$. En esta segunda administración, además, un número significativamente mayor de estudiantes consideró las dificultades de los familiares entre las causas ( $36 \%$ vs. $72 \%, p<.0001)$.

En relación con los prejuicios, en la segunda evaluación, el porcentaje de estudiantes que dijo estar totalmente convencido de que las personas con esquizofrenia eran impredecibles bajó al $6 \%(\mathrm{p}<.0001)$, mientras que la de los estudiantes plenamente convencidos de que estas personas eran peligrosas para otros y para sí mismos resultó ser respectivamente del $8 \%$ y del 17\% $(\mathrm{p}<.0001 ; \mathrm{p}<$. 01 ). Finalmente, la recuperación fue considerada como posible por el $83 \%$ de los estudiantes $(\mathrm{p}<.0001)$.

\section{CONCLUSIONES Y PERSPECTIVAS FUTURAS}

La iniciativa que se describe aquí es la primera realizada en Italia con el objetivo de comprobar si las opiniones de los futuros médicos, sobre los prejuicios más habituales relativos a la esquizofrenia, podrían modificarse a través de una intervención educativa durante el curso de los estudios de Medicina. 
Aunque se trata de una experiencia piloto, que requiere una verificación posterior de sus resultados a través de un estudio clínico controlado y aleatorizado así como una evaluación de la evolución en el tiempo de las variaciones observadas, los resultados son alentadores y en línea con otros estudios sobre este tema (Kassam et al., 2011; Lincoln et al., 2008; Galletly y Burton, 2011). En particular, cabe señalar la significativa variación en la opinión de los estudiantes sobre la imprevisibilidad e incurabilidad, unida una mayor relevancia concedida a los factores psicosociales en la génesis y el curso evolutivo de la esquizofrenia. Es importante señalar, además, que estos cambios no están asociados a un mayor escepticismo sobre los factores biológicos, sino a un equilibrio más claro entre factores biológicos y psicosociales en consonancia con el modelo de vulnerabilidad-estrés de Zubin y Spring (1977).

En tanto que grupo de trabajo compuesto principalmente por estudiantes, creemos que entre los elementos que caracterizan positivamente esta iniciativa figuran: a) el fuerte interés que suscita el tema del estigma y de los derechos al tratamiento entre los estudiantes; b) la disponibilidad de los usuarios a participar activamente en la formación de los futuros médicos, como expertos en su propia situación. La escucha de los testimonios presentados por las personas con trastornos mentales fue evaluada por los participantes como extremadamente útil para reflexionar sobre sus propios comportamientos hacia las personas con trastornos mentales.

Es de esperar que estas iniciativas se conviertan en parte integral del currículo formativo de todos los profesionales sanitarios y contribuyan a mejorar la calidad de la atención y las actitudes hacia las personas con trastornos mentales.

\section{REFERENCIAS}

Angermeyer, M. y Dietrich, S. (2006). Public beliefs about and attitudes towards people with mental illness: a review of population studies. Acta Psychiatrica Scandinavica, 113, 163-179.

Angermeyer, M.C., Holzinger, A., Carta, M. y Schoumerus, G. (2011). Biogenetic explanations and public acceptance of mental illness: systematic review of population studies. British Journal of Psychiatry, 199, 367-372.

Angermeyer, M.C., Holzinger, A. y Matschinger, H. (2010). Emotional reactions to people with mental illness. Epidemiologia e Psichiatria Sociale, 9, 26-32. Arvaniti, A., Samakouri, M., Kalamara, E., Bochtsou, V., Bikos, C., y Livaditis, M. (2009). Health service staff's attitudes towards patients with mental illness. Social Psychiatry and Psychiatric Epidemiology, 44, 658-665.

Ay, P., Save, D. y Fidanoglu, O. (2006). Does stigma concerning mental disorders differ through medical education? A survey among medical students in Istanbul. Social Psychiatry and Psychiatric Epidemiology, 41, 63-67.

Aydin, N., Yigit, A., Inandi, T. y Kirpinar, I. (2003). Attitudes of hospital staff toward mentally ill patients in a teaching hospital, Turkey. International Journal of Social Psychiatry, 49, 17-26. 
Casey, D.E. (2005). Metabolic issues and cardiovascular disease in patients with psychiatric disorders.American Journal of Medicine, 118, 15-22.

Cormack, S. y Furnham, A. (1998). PsychiatricLabelling, sex role stereotypes and beliefs about the mentally ill. International Journal of Social Psychiatry, 44, 235-47.

Dinos, S., Stevens, S., Serfaty, M., Weich, S. y King, M. (2004). Stigma: the feelings and experiences of 46 people with mental illness. Qualitative study. British Journal of Psychiatry,184, 176-81.

Economou, M., Peppou, L.E., Louki, E. y Stefanis, C.N. (2012). Medical students' beliefs and attitudes towards schizophrenia before and after undergraduate psychiatric training in Greece. Psychiatry and Clinical Neurosciences, $66,17-25$.

Fernando, S.M., Deane, F.P. y McLeod, H.J. (2010). Sri Lankan doctors' and medical undergraduates' attitudes towards mental illness. Social Psychiatry and Psychiatric Epidemiology, 45, 733-739.

Galletly, C. y Burton, C. (2011). Improving medical student attitudes towards people with schizophrenia. Australian and New Zealand Journal of Psychiatry, 45, 473-476.

Grausgruber, A., Meise, U., Katschnig, H., Schöny, W. y Fleischhacker, W. (2007). Patterns of social distance towards people suffering from schizophrenia in Austria: a comparison between the general public, relatives and mental health staff. Acta Psychiatrica Scandinavica, 115, 310-319.

Howard, L.M., Barley, E.A., Davies, E. et al. (2010). Cancer diagnosis in people with severe mental illness: Practical and ethical issues. Lancet Oncology, 11, 797-804.

Kassam, A., Glozier, N., Leese, M., Loughran, J. y Thornicroft, G. (2011). A controlled trial of mental illness related stigma training for medical students. BMC Medical Education, 29, 11-51.

Jones, S., Howard, L. y Thornicroft, G. (2008). Diagnostic overshadowing': worse physical health care for people with mental illness. Acta Psychiatrica Scandinavica, 118, 169-173.

Lincoln, T.M., Arens, E., Berger, C. y Rief, W. (2008). Can antistigma campaigns be improved? A test of the impact of biogenetic vs psychosocial causal explanations on implicit and explicit attitudes to schizophrenia. Schizophrenia Bulletin, 34, 984-994.

Llerena, A., Càceres, M., y Peñas-Lledó, E. (2002). Schizophrenia stigma among medical and nursing undergraduates. European Psychiatry, 17, 298-299.

Luchins, D. (2004). At issue: will the term brain disease reduce stigma and promote parity for mental illnesses? Schizophrenia Bulletin, 30, 1043-1048.

Magliano, L., Fiorillo, A., De Rosa, C., Malangone, C. y Maj, M. (2004a). Beliefs about schizophrenia in Italy: A comparative nationwide survey of the general public, mental health professionals, and patients' relatives. Canadian Journal of Psychiatry, 49, 322-330.

Magliano, L., De Rosa, C., Fiorillo, A., et al. (2004b). Beliefs of psychiatric 
nurses about schizophrenia: A comparison with patients' relatives and psychiatrists. International Journal of Social Psychiatry, 50, 319-330.

Magliano, L., Fiorillo, A., Malangone, C., Del Vecchio, H. y Maj, M. (2008). Users' Opinions Questionnaire Working Group. Views of persons with schizophrenia on their own disorder: An Italian participatory study. Psychiatric Services, 59, 795-799.

Magliano, L., Read, J., Rega, S., Oliviero, N., Sagliocchi, A., Patalano, M., y D'Ambrosio, A. (2011). The influence of causal explanations and diagnostic labeling on medical students' views of schizophrenia. Academic Medicine, 86, 1155-1162.

Magliano, L., Read, J., Sagliocchi, A., Patalano, M., D’Ambrosio, A. y Oliviero, N. (in press). Differences in views of schizophrenia during medical education: a comparative study of 1st vs. 5th-6th year Italian medical students. Social Psychiatry and Psychiatric Epidemiology.

Naeem, F., Ayub, M., Javed, Z., Irfan, M., Haral, F. y Kingdon, D. (2006) Stigma and psychiatric illness. A survey of attitude of medical students and doctors in Lahore. Pakistan. Journal of Ayub Medical College Abbottabad, 18, 46-49.

Newcomer, J.W. (2007). Antipsychotic medications: metabolic and cardiovascular risk. Journal of Clinical Psychiatry, 68, 8-13.

Ng, R., Pearson, V., Chen, E., y Law, C. (2011). What does recovery from schizophrenia mean? Perceptions of medical students and trainee psychiatrists. International Journal of Social Psychiatry, 57, 248-262.

Nordt, C., Rössler, W. y Lauber, C. (2006). Attitudes of mental health professionals toward people with schizophrenia and major depression. Schizophrenia Bulletin, 32, 709-714.

Ogunsemi, O.O., Odusan, O. y Olatawura, M.O. (2008). Stigmatising attitude of medical students towards a psychiatry label. Annals of General Psychiatry, $25,7-15$.

Patel, M. (2004). Attitudes to psychosis: health professionals. Epidemioliologiae Psichiatria Sociale, 13, 213-218.

Pescosolido, B., Martin, J., Long, J., Medina, T., Phelan, J., y Link, B. (2010). "A desease like any other?" A decade of change in public reactionsto schizophrenia, depression, and alcohol dependance. The American Journal of Psychiatry, 167, 1321-1330.

Read, J., Haslam, N., Magliano, L. (in press). Prejudice, stigma and 'schizophrenia' The role of bio-genetic ideology. In J Read, J Dillon (eds.) Models of Madness (2nd edition). London: Routledge.

Read, J., Haslam, N., Sayce, L. y Davies, E. (2006). Prejudice and schizophrenia: A review of the 'mental illness is an illness like any other'approach. Acta Psychiatrica Scandinavica, 114, 303-318.

Sartorius, N. (2002). Iatrogenic stigma of mental illness: Begins with behaviour and attitudes of medical professionals, especially psychiatrists. British Medical Journal, 324, 1470-1471.

Schulze, B. y Angermeyer, M.C. (2003). Subjective experiences of stigma. A 
focus group study of schizophrenic patients, their relatives, and mental health professionals. Social Sciences and Medicine, 56, 299 -312.

The Lancet (2001). No mental health without physical health. The Lancet, 377, 611.

Thornicroft, G., Rose, D., y Kassam, A. (2007). Discrimination in health care against people with mental illness. International Review of Psychiatry, 19, 113-22.

Wahl, O. (1999). Mental health consumers' experience of stigma. Schizophrenia Bulletin, 25, 467-478.

Zubin, J. y Spring, B. (1977). Vulnerability-A new view of schizophrenia. Journal of Abnormal Psychology, 86, 103-126.

\section{MATERIALES UTILIZADOS EN LA ELABORACIÓN DEL CURSO}

\section{Video}

http://www.youtube.com/watch?v=5Op7INlzM5M

http://news.bbc.co.uk/2/hi/uk_news/8192603.stm

http://bringchange2mind.org/

\section{Artículos}

Bellack, A. (2006). Scientific and consumer models of recovery in schizophrenia: concordance, contrasts, and implications. Schizophrenia Bulletin, 32, 432-442.

Chi ruba i bambini? I risultati di uno studio sui casi di presunto tentato rapimento di minori da parte di rom riferiti in Italia dal 1986 al 2007 http://www.personaedanno.it/attachments/allegati_articoli/AA_018736_resource1_orig.pdf

Fazel, S., Långström, N., Hjern, A., Grann, M. y Lichtenstein, P. (2009). Schizophrenia, substance abuse, and violent crime. JAMA, 301, 2016-2023.

Harrison, G., Hopper, K., Craig, T., Laska, E., Siegel, C., Wanderling, J., Dube, K.C., Ganev, K., Giel, R., An Der Heiden, W., Holmberg, S.K., Janca, A., Lee, P.W.H., León, C.A., Malhotra, S., Marsella, A.J., Nakane, Y., Sartorius, N., Shen, Y., Skoda, C., Thara, R., Tsirkin, S.J., Varma, V.K., Walsh, D. y Wiersma, D. (2001). Recovery from psychotic illness: a 15- and 25-year international follow-up study. British Journal of Psychiatry, 178, 506-517.

Harrow, M., Grossman, L.S., Jobe, T.H., Herbener, E.S. (2005). Do patients with schizophrenia ever show periods of recovery? A 15-year multi-follow-up study. Schizophrenia Bulletin, 31, 723-734.

Jablenski, A. y Sartorius, N. (2008). What did the WHO studies really find? Schizophrenia Bulletin, 34, 253-255.

Large, M., Smith, G. y Nielssen, O. (2009). The relationship between the rate of homicide by those with schizophrenia and the overall homicide rate: a systematic review and meta-analysis. Schizophrenia Research, 112, 123-129. 
Large, M., Smith, G., Swinson, N., Shaw, J. y Nielssen, O. (2008). Homicide due to mental disorder in England and Wales over 50 years. British Journal of Psychiatry, 193, 130-133.

Liberman, R. y Kopelowitz, A. (2005). Recovery from schizophrenia:a concept in search of research. Psychiatric Services, 56, 735-742

The National Consortium on Stigma and Empowerment http://www.stigmaandempowerment.org/

Tibaldi, G. y Govers, L. (2009). Evidence-based hope. La proposta di una prospettiva comune. Psichiatria di Comunità, 3, 117-128.

Troppi pregiudizi sui nomadi. Non è vero che rapiscono i bambini http://www. repubblica.it/2008/11/sezioni/cronaca/rom-rapimenti/rom-rapimenti/romrapimenti.html?ref=search

Warner, R. (1994). Recovery from schizophrenia: Psychiatry and political economy. Routledge: London. 